\title{
An Improved Particle Swarm Optimization Algorithm Using Eagle Strategy for Power Loss Minimization
}

\author{
Hamza Yapıcı ${ }^{1}$ and Nurettin Çetinkaya ${ }^{2}$ \\ ${ }^{1}$ The Graduate School of Natural and Applied Science, Selçuk University, Konya, Turkey \\ ${ }^{2}$ Electrical \& Electronics Engineering Department, Selçuk University, Konya, Turkey \\ Correspondence should be addressed to Hamza Yapıc1; hyapici@konya.edu.tr
}

Received 12 January 2017; Accepted 15 March 2017; Published 30 March 2017

Academic Editor: Blas Galván

Copyright ( 2017 Hamza Yapıcı and Nurettin Çetinkaya. This is an open access article distributed under the Creative Commons Attribution License, which permits unrestricted use, distribution, and reproduction in any medium, provided the original work is properly cited.

\begin{abstract}
The power loss in electrical power systems is an important issue. Many techniques are used to reduce active power losses in a power system where the controlling of reactive power is one of the methods for decreasing the losses in any power system. In this paper, an improved particle swarm optimization algorithm using eagle strategy (ESPSO) is proposed for solving reactive power optimization problem to minimize the power losses. All simulations and numerical analysis have been performed on IEEE 30-bus power system, IEEE 118-bus power system, and a real power distribution subsystem. Moreover, the proposed method is tested on some benchmark functions. Results obtained in this study are compared with commonly used algorithms: particle swarm optimization (PSO) algorithm, genetic algorithm (GA), artificial bee colony (ABC) algorithm, firefly algorithm (FA), differential evolution (DE), and hybrid genetic algorithm with particle swarm optimization (hGAPSO). Results obtained in all simulations and analysis show that the proposed method is superior and more effective compared to the other methods.
\end{abstract}

\section{Introduction}

The reactive power optimization approach is important for power quality, system stability, and optimal operation of electrical power systems. Reactive power control can be set with adjusting the voltage levels, tap positions of transformers, shunt capacitors, and other control variables. The reactive power optimization approach can minimize the power losses and improve the voltage profiles. Many conventional methods such as dynamic programming, linear and nonlinear programing, interior point method, genetic algorithm, and quadratic programming have been employed for solving reactive power optimization problem [1-5].

Moreover, in the last years, various intelligence computation methods have proposed for the reactive power optimization such as particle swarm optimization, differential evolution, ant colony, and $\mathrm{BC}$ [6-9]. The optimization methods along with fuzzy logic [10] have been used to adjust the optimal setting of power system variables, containing flexible AC transmission systems (FACTs) devices, where the power system losses have been reduced by the optimal placement of thyristor-controlled series compensation (TCSC) and static VAR compensator (SVC). A dynamic weights based particle swarm optimization (PSO) algorithm has been used for reducing power loss [11]. This approach has been implemented to IEEE 6-bus system. The PSO based reactive power optimization method has been presented in [12] for minimizing the total support cost from generators and reactive compensators. In [13], modified artificial fish swarm algorithm (MAFSA) has been proposed to optimize the reactive power optimization and this method has been applied to IEEE 57-bus system. A seeker optimization algorithm has been presented for reactive power dispatch method in [14]; the authors applied the algorithm to several benchmark functions, IEEE 57 and IEEE 118 test systems and then compared with different conventional nonlinear programming methods (such as different versions of GA, DE, and PSO). The optimum conditions for operating the electric power systems have been determined by GA [15]. Authors selected the main objective of the study as the definition of the load buses 
voltage amplitude values. By this way, they get the minimum power losses in the transmission lines. In [16], reactive power optimization has been solved by adjusting generator voltages, transformer taps, and capacitors/reactors and three GA/SA/TS hybrid algorithms have been used. In the other study [17], the reactive power optimization problem has been solved by using evolutionary computation techniques such as genetic and particle swarm optimization algorithms, and voltage bus magnitude, transformer tap setting, and the reactive power injected by capacitor banks were selected as control variables. Authors applied the proposed algorithms to IEEE 30-bus and IEEE 118-bus systems. In [18], distributed generation has been taken into distribution system and a multiobjective model for reactive power optimization has been investigated to reduce the system loss and voltage deviation and minimize the total reactive compensation devices capacity. Simulation and analysis have been carried out on IEEE 33bus system with a dynamically adaptive multiobjective particle swarm optimization (DAMOPSO) algorithm. In [19], an improved mind evolutionary algorithm (IMEA) has been presented for optimal reactive power dispatch and voltage control. The proposed method has been carried out on IEEE 30-bus system and simulation results have been compared with GA to prove its efficiency and superiority. On the other study, GA has been proposed for optimization of reactive power flow in a power system [20]. The authors used two different objective functions: active power losses and the voltage-stability-oriented index.

In this study, a particle swarm optimization algorithm using eagle strategy (ESPSO) has been developed to implement reactive power optimization for reducing power losses. Eagle strategy (ES) has been originated by the foraging behavior of eagles such as golden eagles. This strategy has two important parameters: random search and intensive chase. At first it explores the search space globally, and then in the second case the strategy makes an intensive local search with using an effective local optimizer method [21-23]. So, SPO has been improved using ES and implemented to reactive power optimization problem.

Moreover, simulations and analysis of reactive power optimization problem have been performed on IEEE 30-bus test system, IEEE 118-bus test system, and a real power subsystem, and the proposed approach has been compared with various algorithms to show performance. It can be seen that, in case studies, the proposed approach has been outperformed compared to other methods mentioned.

\section{Problem Formulation}

This paper defines the objective function as reducing power losses of power system. The objective function given in [14, $15,17,24]$ for reactive power optimization problem is given in (1). The equality and inequality constraints are denoted in (3). Note that the real distribution subsystem considered in this paper has no generators; therefore, we did not handle the generator constraints for this power system. Moreover, control variables are self-constrained but dependent variables are implemented to the objective function by using penalty terms. So, the objective function can be given as in (2) as follows:

$$
\begin{aligned}
& P_{\text {Loss }}=\sum_{i=1}^{N_{i}}\left[g_{h(i, j)} \cdot\left(V_{i}^{2}+V_{j}^{2}-2 \cdot V_{i} \cdot V_{j} \cdot \cos \left(\theta_{i, j}\right)\right)\right], \\
& f(\vec{x}, \vec{y}) \\
& =P_{\text {Loss }}+\lambda_{1} \sum_{N_{V}} \Delta{V_{L}}^{2}+\lambda_{2} \sum_{N_{Q}} \Delta Q_{G}^{2}+\lambda_{3} \sum_{N_{i}} \Delta I_{l(i, j)}{ }^{2},
\end{aligned}
$$

where $\lambda_{1}, \lambda_{2}$, and $\lambda_{3}$ are the penalty factors which are equal to $1000, N_{V}$ is the number of load buses, and $N_{Q}$ is the number of generator buses which injected reactive power. If $V_{L}<V_{L \text { min }}$ then $\Delta V_{L}$ is equal to $V_{L \text { min }}-V_{L}$ and if $V_{L \max }<V_{L}$ then $\Delta V_{L}$ is equal to $V_{L}-V_{L \max }$. On the other hand, if $Q_{G}<Q_{G \min }$ then $\Delta Q_{G}$ is equal to $Q_{G \min }-Q_{G}$ and if $Q_{G \max }<Q_{G}$ then $\Delta Q_{G}$ is equal to $Q_{G}-Q_{G \max }$. If $I_{l(i, j)}>I_{l(i, j) \max }$ then $I_{l(i, j)}$ is equal to $I_{l(i, j) \max }$.

Note that superscripts "min" and "max" in (3) express lower and upper limits, respectively. Here, $\vec{x}$ is the vector of control variable where $\vec{x}=\left[\begin{array}{lll}V_{G} & T_{K} & Q_{C}\end{array}\right]^{T}$ and $\vec{y}$ is the vector of dependent variable where $\vec{y}=\left[\begin{array}{lll}V_{L} & Q_{G} & I_{l}\end{array}\right]^{T}$.

$$
\begin{aligned}
& P_{G i}-P_{l i}-V_{i} \\
& \quad \cdot \sum_{j \in N i}\left[V_{j} \cdot\left(g_{h(i, j)} \cdot \cos \left(\theta_{(i, j)}\right)+b_{h(i, j)} \cdot \sin \left(\theta_{(i, j)}\right)\right)\right] \\
& \quad=0, \quad i \in N_{\mathrm{slb}}, \\
& Q_{G i}-Q_{l i}-V_{i} \\
& \quad \cdot \sum_{j \in N i}\left[V_{j} \cdot\left(g_{h(i, j)} \cdot \sin \left(\theta_{(i, j)}\right)-b_{h(i, j)} \cdot \cos \left(\theta_{(i, j)}\right)\right)\right] \\
& \quad=0, \quad i \in N_{\mathrm{pq}}, \\
& T_{K \min } \leq T_{K} \leq T_{K \max }, \quad K \in N_{K}, \\
& Q c_{i \min } \leq Q_{i} \leq Q_{c_{i \max }}, \quad i \in N_{C}, \\
& P_{G i \min } \leq P_{G i} \leq P_{G i \max }, \quad i \in N_{G}, \\
& Q_{G i \min } \leq Q_{G i} \leq Q_{G i \max }, \quad i \in N_{G}, \\
& V_{i \min } \leq V_{i} \leq V_{i \max }, \quad i \in N_{i}, \\
& I_{l(i, j)}<I_{l(i, j) \max }, \quad i, j \in N_{i} .
\end{aligned}
$$

\section{Method}

3.1. Eagle Strategy(ES). Eagle strategy (ES) is a two-stage process, developed by Yang et al. [22]. ES is inspired by the foraging behavior of eagles that they fly random in analogy to the Lévy flights. It uses different algorithms which make global search and local search for fitting different proposes. ES has some similarities with random restart hill climbing method, but there are two important differences: ES is a two-stage method, and so a global search randomization method and an intensive local search are combined and ES uses Lévy walks so it can explore the global search space more effectively. 


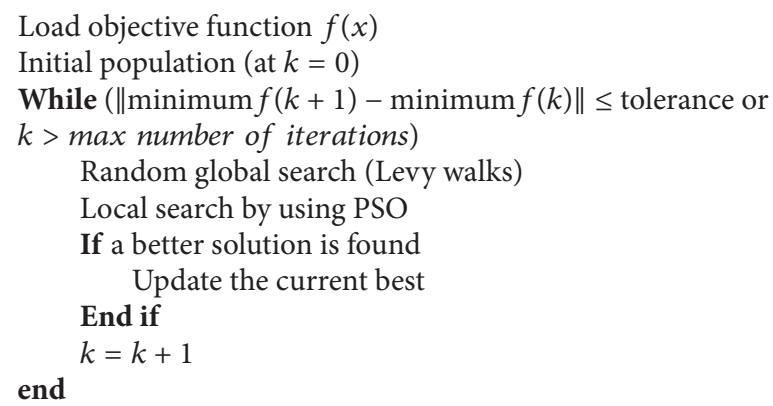

Pseudocode 1

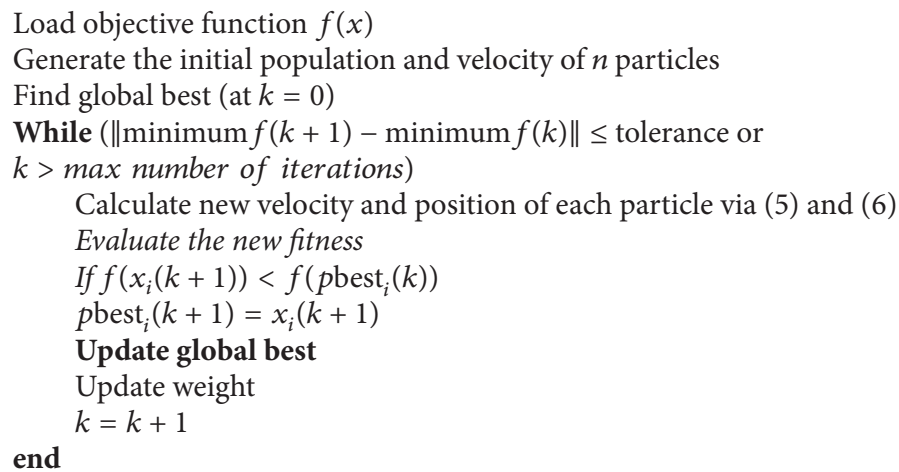

Pseudocode 2

Essentially, ES makes the global search in the $n$-dimensional space with Lévy flights; if any probable solution is found, an intensive local optimizer is put to use for local search such as differential evolution, particle swarm optimization algorithm, and artificial bee colony that these have local search capability. Then the procedure starts again with new global search in the new area [21-23, 25, 26].

Note that ES is not an algorithm; it is a method. In fact, various algorithms can be used at the different stages. This provides that it combines the advantages of these different algorithms so as to obtain better results.

Lévy distribution [22] is given as follows:

$$
\begin{aligned}
L(s) \sim \frac{\lambda \Gamma(\lambda) \sin (\pi \lambda / 2)}{\pi} \frac{1}{s^{1+\lambda}}, & \\
& \left(s \gg s_{0}>0\right),(1<\lambda \leq 3),
\end{aligned}
$$

where $\Gamma(\lambda)$ is standard gamma function and $s$ is the step length. When $\lambda=3$, it becomes Brownian motion as a special case. We used $\lambda=2$; thus Lévy walks become the Cauchy distribution.

Pseudocode of ES can be given as in Pseudocode 1.

3.2. Particle Swarm Optimization (PSO). This technique processing is so easy that PSO utilizes some parameters and definitions of the optimization process and then it starts the process with an initial random population, named particles.
Each of these particles has a possible solution for the main problem and is processed as a part in $n$-dimensional space. In the variable space, each particle has a position identified by $x_{i}^{k}=\left(x_{i 1}^{k}, x_{i 2}^{k}, \ldots, x_{i n}^{k}\right)$ and a velocity identified by $v_{i}^{k}=$ $\left(v_{i 1}^{k}, v_{i 2}^{k}, \ldots, v_{i n}^{k}\right)$. Velocity and position of each particle is updated using (5) and (6), respectively. If a particle has a best position, it is carried to the next. Additionally, best positions are represented as pbest and the best position of all particles is represented as gbest [27-29].

$$
\begin{aligned}
& v_{i}^{k+1}=w_{i} v_{i}^{k}+c_{1} r_{1}\left(\text { pbest }_{i}-x_{i}^{k}\right)+c_{2} r_{2}\left(\text { g best }_{i}-x_{i}^{k}\right), \\
& x_{i}^{k+1}=x_{i}^{k}+v_{i}^{k+1} .
\end{aligned}
$$

The weight function is

$$
w_{i}=w_{\max }-\frac{w_{\max }-w_{\min }}{k_{\max }} \times k,
$$

where $r_{1}$ and $r_{2}$ are selected randomly in the range $[0,1]$ and $c_{1}$ and $c_{2}$ are acceleration coefficients that inspect the motion of a particle.

$$
\text { Pseudocode of PSO see Pseudocode } 2 .
$$

3.3. Eagle Strategy with Particle Swarm Optimization (ESPSO). We know that for local search we can use an algorithm such as PSO, DE, and ABC; therefore, PSO is applied to the local 
Step 1. Load function and its parameters

Step 2. Generate initial population randomly

Step 3. While $\|\operatorname{minimum} f(k+1)-\operatorname{minimum} f(k)\| \leq$ tolerance or

$k>$ max number of iterations,

performing random global search using Levy Flight $x^{k+1}=x^{k}+\alpha L(s, \lambda)$,

$(\lambda=1.5, \alpha=1$, and step length $s$ set as $s=5)$

Then, find a promising solution

Step 4. Determine a random number. Set switching parameter $p$ for controlling

between global search and local search. (We set $p=0.2$ )

If $p<$ rand else

switch to local search stage (go to Step 5)

switch to global search stage (go to Step 6)

Step 5. In intensive local search stage, search around a promising solution, Calculate new velocity and position of each particle via (5) and (6), Then evaluate new fitness (Use the objective function based on Newton-Raphson power flow for reactive power optimization problem)

If $f\left(x_{i}(k+1)\right)<f\left(\right.$ pbest $\left._{i}(k)\right)$

Step 6. Update, pbest $_{i}(k+1)=x_{i}(k+1)$

$k=k+1$

Step 7. Stopping criterion, Maximum number of iterations or a given tolerance (tolerance set as $1.0000 e-9$ for reactive power optimization problem)

Step 8. If any criterion is provided, then stop the algorithm else go to Step 3

Algorithm 1

search stage of ES method. On the other side, randomization with Lévy walks can be used in the global search. The proposed method is a population-based algorithm.

We used the parameters of PSO used in most applications $[18,19]$, where $c_{1}=c_{2}=2, w_{\min }=0.4$, and $w_{\max }=0.9$; then we set $\lambda=2$. We used 500 iterations for benchmark functions and 100 iterations for reactive power optimization problem. There are two important situations, $\Gamma \rightarrow \infty$ and $\Gamma \rightarrow 0$. If $\Gamma \rightarrow \infty$, the velocity of particles cannot be decreased and particles are far from one another. If $\Gamma \rightarrow 0$, then the particles are short sighted, so particles will be trapped in a confined space and velocity of this particles can be very small.

There are several stopping criteria given in the literature: a fixed number of generations, the number of iterations since the last change of the best solution being greater than a specified number, the number of iterations reaching maximum number, a located string with a certain value, and no change in the average fitness after some generations. In this paper, the stopping criteria are chosen as the maximum number of iterations and the tolerance value for fitness where $\|\operatorname{minimum} f(k+1)-\operatorname{minimum} f(k)\| \leq$ tolerance.

Steps of proposed method have been explained in Algorithm 1.

\section{Performance of ESPSO on Benchmark Functions}

For testing proposed method, seven well-known benchmark functions are handled with comparison of widely used six algorithms (GA, PSO, ABC, FA, and hGAPSO). These benchmark functions are listed in Table 1.

All the methods run in 500 iterations and over 50 times for each function. The results obtained by proposed ESPSO algorithm on some benchmark functions are statistically different from the other algorithms and ESPSO has a good performance on the test functions. Results point out that ESPSO algorithm is appropriate for optimizations of unimodal and multimodal functions. Table 2 shows the results obtained by all methods mentioned in this section. In Table 2, it can be seen that the proposed approach has obtained better results than the other methods.

Unlike other algorithms, ESPSO performs global search and local search by using parameter $p$ until converging to optimum as possible. In addition, it generates initial population randomly using uniform distribution function in MATLAB. Consequently, from the local search to global search, fitness value is changed from the worst to the best, so as a generation can not only find a good search but also switch to new search area in the search space.

\section{Implementing Reactive Power Optimization}

In reactive power optimization problem, transformer tap positions and shunt capacitor banks are discrete or integer variables. However, the proposed method only uses continuous variable. To use these variables, each particle of ESPSO explores in the space as searching for continuous variable, and objective function is evaluated by cutting the corresponding dimensions of particles into integers. This means that the 
TABLE 1: Benchmark functions.

\begin{tabular}{|c|c|c|c|}
\hline Equation & Name & $D$ & Feasible bounds \\
\hline$f_{1}=\sum_{i=1}^{D} x_{i}^{2}$ & Sphere/parabola & 30 & $(-100,100)^{D}$ \\
\hline$f_{2}=-20 \exp \left\{-0.2 \sqrt{\frac{1}{D} \sum_{i=1}^{D} x_{i}^{2}}\right\}-\exp \left\{\frac{1}{D} \sum_{i=1}^{D} \cos \left(2 \pi x_{i}\right)\right\}+20+e$ & Ackley & 30 & $(-32,32)^{D}$ \\
\hline$f_{3}=\sum_{i=1}^{D-1}\left\{100\left(x_{i+1}-x_{i}^{2}\right)^{2}+\left(x_{i}-1\right)^{2}\right\}$ & Rosenbrock & 30 & $(-30,30)^{D}$ \\
\hline$f_{4}=\frac{1}{4000} \sum_{i=1}^{D} x_{i}^{2}-\prod_{i=1}^{D} \cos \left(\frac{x_{i}}{\sqrt{i}}\right)+1$ & Generalized Griewank & 30 & $(-600,600)^{D}$ \\
\hline$f_{5}=-\sum_{i=1}^{4} c_{i} \exp \left[-\sum_{j=1}^{\infty} a_{i j}\left(x_{j}-p_{i j}\right)^{2}\right]$ & Hartman 3 & 3 & $(0,1)^{D}$ \\
\hline$f_{6}=4 x_{1}^{2}-2.1 x_{1}^{4}+\frac{1}{3} x_{1}^{6}+x_{1} x_{2}-4 x_{2}^{2}+4 x_{2}^{4}$ & Six-hump camel-back & 2 & $(-5,5)^{D}$ \\
\hline $\begin{array}{l}f_{7}=\left\{1+\left(x_{1}+x_{2}+1\right)^{2}\left(19-14 x_{1}+3 x_{1}^{2}-14 x_{2}+6 x_{1} x_{2}+3 x_{2}^{2}\right)\right\} \times \\
\left\{30+\left(2 x_{1}-3 x_{2}\right)^{2}\left(18-32 x_{1}+12 x_{1}^{2}+48 x_{2}-36 x_{1} x_{2}+27 x_{2}^{2}\right)\right\}\end{array}$ & Goldstein-Price & 2 & $(-2,2)^{D}$ \\
\hline
\end{tabular}

TABLE 2: Comparison of ESPSO with other methods on test functions.

\begin{tabular}{|c|c|c|c|c|c|c|c|}
\hline Function & Index & GA & PSO & $\mathrm{ABC}$ & FA & hGAPSO & ESPSO \\
\hline \multirow{2}{*}{$f_{1}$} & Best & 1.1091 & 0.9206 & 0.8991 & 0.0037 & 0.8874 & 0.0026 \\
\hline & Mean & 1.7623 & 1.0038 & 1.2978 & 0.0126 & 1.1172 & 0.9032 \\
\hline \multirow{2}{*}{$f_{2}$} & Best & 0.9398 & 0.7646 & 0.8991 & 0.0317 & 0.0918 & 0.0125 \\
\hline & Mean & 1.6693 & 1.6237 & 2.6842 & 0.1004 & 1.9044 & 1.0005 \\
\hline \multirow{2}{*}{$f_{3}$} & Best & 101.9834 & 24.0076 & 38.5673 & 17.2783 & 22.9814 & 16.5622 \\
\hline & Mean & 217.0324 & 65.3482 & 94.0345 & 19.2953 & 28.0912 & 54.8731 \\
\hline \multirow{2}{*}{$f_{4}$} & Best & 0.1983 & 0.0078 & 0.0659 & $9.9047 e-04$ & $1.1092 e-3$ & $3.4597 e-4$ \\
\hline & Mean & 0.9219 & 0.0396 & 0.15415 & 0.0117 & 0.0335 & 0.1931 \\
\hline \multirow{2}{*}{$f_{5}$} & Best & -3.8664 & -3.8642 & -3.8652 & -3.8628 & -3.8629 & -3.8628 \\
\hline & Mean & -3.8824 & -3.8659 & -3.8739 & -3.8656 & -3.8728 & -3.8638 \\
\hline \multirow{2}{*}{$f_{6}$} & Best & -1.0525 & -1.0393 & -1.0353 & -1.0317 & -1.0318 & -1.0316 \\
\hline & Mean & -1.1316 & -1.0626 & -1.0445 & -1.0319 & -1.0336 & -1.0330 \\
\hline \multirow{2}{*}{$f_{7}$} & Best & 3.1870 & 3.0782 & 3.0390 & 3.0256 & 3.0280 & 3.0130 \\
\hline & Mean & 3.7683 & 3.5900 & 3.4802 & 3.3380 & 3.4517 & 3.2300 \\
\hline
\end{tabular}

real position values of particles consist of values of capacitor banks and transformers tap positions represented as a vector to be used for calculating objective function.

Here, inequality constraints are handled in iterations as follows: if variables violate their limit, they are clamped to their upper limit and the remaining mismatch is taken by another one not on limit based on their inertia, and during computation if any PV bus reactive power is violated then PV bus is assumed as PQ bus fixing at the brink value.

Furthermore, the procedure of solving reactive power problem can be explained as follows.

The proposed approach of ESPSO loads parameters of power system and initial condition and specifies upper and lower limits. Initial particles are determined randomly via uniform distribution to locate their initial positions, and initial velocities of particles are constituted. The algorithm evaluates the fitness using objective function and adjusts local bests and global best from locals.
While stopping criteria are provided, ESPSO performs that searching global best by Lévy flight and then determining a random number. If this determined random number is less then $p$ parameter, local search would be performed and locals would be updated via local search property of PSO (update velocities and positions of particles). Then, new fitness computed and if fitness found in local search stage is less then fitness found in first stage, global solution and positions of particles would be updated: $(f(k+1) \leftarrow f(k)$, pbests $(k+1) \leftarrow x(k)$, and then $k=k+1)$.

\section{Results of Simulations}

To verify the ability, capability, and performance of the proposed ESPSO on reactive power optimization problem, it is implemented to IEEE 30-bus test system, IEEE 118-bus test system, and a real distribution subsystem. For testing and 
TABLE 3: Limits of variables (pu).

\begin{tabular}{lcccccc}
\hline Bus & 1 & 2 & 5 & 8 & 11 \\
\hline$P_{G \min }$ & 0.500 & 0.200 & 0.150 & 0.100 & 0.100 & 0.120 \\
$P_{G \max }$ & 2.000 & 0.800 & 0.500 & 0.350 & -0.150 & -0.300 \\
$Q_{G \min }$ & -0.200 & -0.200 & -0.150 & 0.600 & 0.400 \\
$Q_{G \max }$ & 2.000 & 1.000 & 0.800 & $T_{K \max }$ & 0.500 & $Q_{C \min }$ \\
\hline Variable & $V_{\min }$ & $V_{\max }$ & 1.10 & 0.600 \\
Limit & 0.90 & 1.10 & 0.90 & $Q_{C \max }$ \\
\hline
\end{tabular}

TABLE 4: Control variables values and power loss (control variables given pu).

\begin{tabular}{lccccccc}
\hline Variables & GA & PSO & ABC & FA & DE & hGAPSO & ESPSO \\
\hline$V_{1}$ & 1.0345 & 1.0606 & 1.0927 & 1.1000 & 1.0500 & 1.0300 & 1.0770 \\
$V_{2}$ & 1.0463 & 1.0524 & 1.0880 & 1.0967 & 1.0446 & 1.0400 & 1.0775 \\
$V_{5}$ & 1.0294 & 1.0284 & 1.0695 & 1.0850 & 1.0247 & 1.0700 \\
$V_{8}$ & 1.0283 & 1.0289 & 1.0722 & 1.0895 & 1.0265 & 1.0200 & 1.0700 \\
$V_{11}$ & 1.0517 & 0.9833 & 1.0860 & 1.0930 & 1.1000 & 1.0300 & 1.0800 \\
$V_{13}$ & 1.0214 & 0.9924 & 1.0926 & 1.0969 & 1.1000 & 0.9500 & 1.0810 \\
$T_{K(6-9)}$ & 1.0314 & 1.0530 & 0.9983 & 1.0478 & 1.0000 & 1.0300 & 1.0050 \\
$T_{K(6-10)}$ & 1.0994 & 1.1000 & 0.9994 & 0.9439 & 1.1000 & 1.0800 \\
$T_{K(4-12)}$ & 1.1000 & 1.0745 & 0.9984 & 1.0318 & 1.0800 & 1.7000 \\
$T_{K(28-27)}$ & 0.9071 & 0.9247 & 1.0034 & 1.0044 & 0.9200 & 1.0050 \\
$Q_{\text {C10 }}$ & 0.0153 & 0.1174 & 0.0155 & 0.0534 & 0.2600 & 0.9800 \\
$Q_{\text {C24 }}$ & 0.0063 & 0.0056 & 0.0371 & 0.0663 & 0.1000 & - \\
$P_{\text {loss }}(\mathrm{MW})$ & 4.78 & 4.39 & 3.09 & 4.71 & 5.01 & 0.9720 \\
\hline
\end{tabular}

proving the performance of proposed method, it is compared with various algorithms such as GA, PSO [17], ABC [24], FA [30], DE [31], and hGAPSO [32].

The population-based algorithms GA, PSO, DE, and their improved version have a great interest in engineering optimization problems and they all have been successfully implemented to reactive power optimization problems $[4,6$, $7,11,12,16,17,31,32]$. In some other studies, ABC and FA $[24,30]$ have also been used for reactive power optimization. So, ESPSO is compared with these algorithms.

In [17], authors proposed GA and PSO to solve reactive power optimization problem for some test systems where, on IEEE 30-bus system, they obtained power losses $4.78 \mathrm{MW}$ and 4.39 MW with GA and PSO, respectively. In [24], authors obtained power losses with $\mathrm{ABC}$ in the study for 30-bus system as $3.09 \mathrm{MW}$. In a different study for IEEE 30-bus power system, power loss has been tabulated as $4.71 \mathrm{MW}$ with FA [30]. Authors considered four different test systems with various algorithm and they obtained power losses for 30-bus system as $5.01 \mathrm{MW}$ with DE [31]. In [32] minimum power losses have been tabulated as 3.69 MW for IEEE 30-bus test system. (Note that data and settings of all methods can be found in these studies.)

The results of studies in [17] with GA and PSO, [24] with ABC, [31] with DE, and [32] with hGAPSO are taken into consideration for IEEE 118-bus test system. In these studies, authors obtained power losses with GA, PSO, ABC, and DE as $139.16,135.64,119.6923$, and 128.318 , respectively.
The proposed method is coded in MATLAB software for 30-bus and 118-bus test systems. Maximum number of iterations is set as 100 and ESPSO is run over 50 times. The best solutions for both systems are tabulated after 50 times running.

6.1. IEEE 30-Bus Test System. Power system includes 41 branches, 6 generators, 21 load buses, and 9 shunt compensators. Branches of 4-12, 6-9, 6-10, and 28-27 are adjustable tab under load and symbolized as $T_{K(4-12)}, T_{K(6-9)}, T_{K(6-10)}$, and $T_{K(28-27)}$, respectively. All data and parameters of power system used can be found in [33]. The system loads are as follows: $P_{\text {load }}=2.834 \mathrm{pu}$ and $Q_{\text {load }}=1.262 \mathrm{pu}$. The transformers tap settings and bus voltages lower and upper limits are 0.9 pu and $1.1 \mathrm{pu}$ and shunt compensators limits are $0.0 \mathrm{pu}$ and $0.05 \mathrm{pu}$, respectively, that variables limits are denoted in Table 3.

For comparing the proposed approach with other algorithms mentioned previously, index related to performance consists of the minimization of power loss given in Table 4. Table 4 shows that ESPSO found system loss less than other techniques, so that it can be deduced that the proposed method is clearly more robust and better than others in this case study. Variables values obtained by solving reactive power optimization problem for IEEE 30-bus system are also illustrated in Table 4. The power loss is reduced to $3.0128 \mathrm{MW}$ with ESPSO, which is less than that obtained by the other methods. On the other hand, the bus voltages are kept 
TABLE 5: Generators powers (MW).

\begin{tabular}{lc}
\hline Variables & ESPSO \\
\hline$P_{G 1}$ & 51.50 \\
$P_{G 2}$ & 80.00 \\
$P_{G 5}$ & 50.00 \\
$P_{G 8}$ & 35.00 \\
$P_{G 11}$ & 30.00 \\
$P_{G 13}$ & 39.98 \\
\hline
\end{tabular}

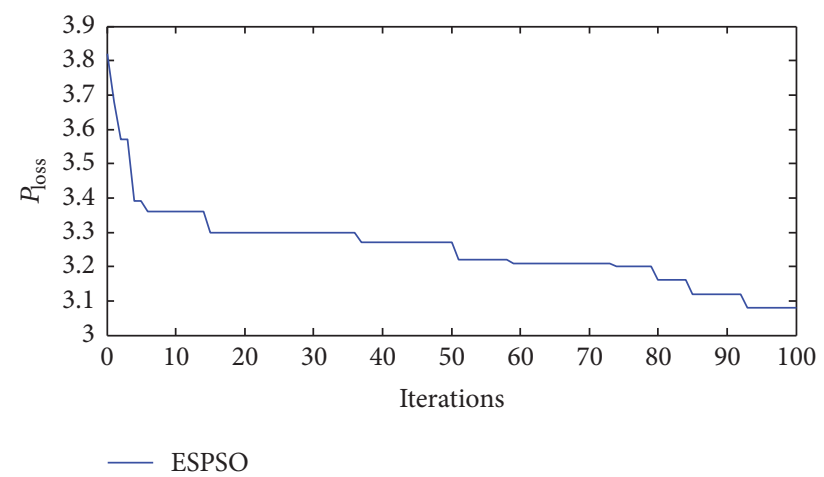

FIgURE 1: The convergence curve of power loss (MW) for 30-bus power system.

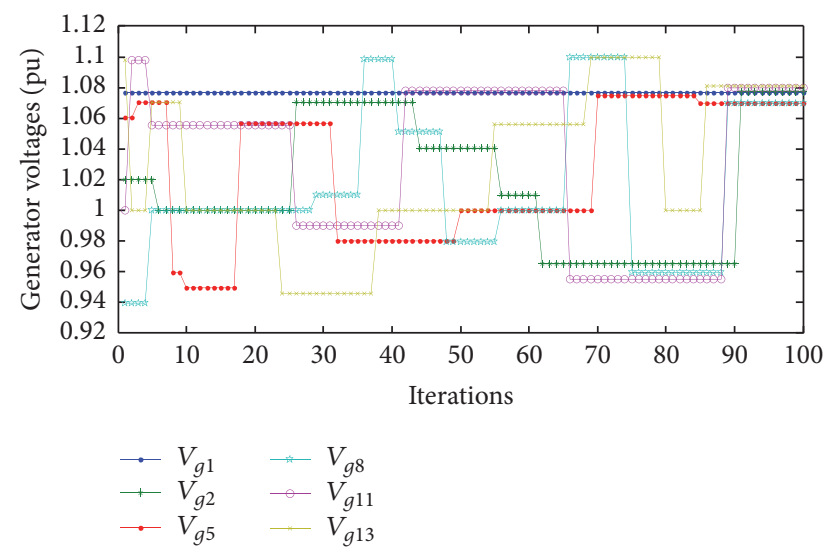

FIGURE 2: The variation of generator voltages for IEEE 30-bus power system.

within their limits; it shows that the proposed method has a best performance for optimizing voltage quality. Outputs of generators are denoted in Table 5 and the variation of power loss obtained by proposed approach is also given in Figure 1. Furthermore, the variation graphs of control variables are illustrated in Figures 2-4.

6.2. IEEE 118-Bus Test System. The IEEE 118-bus system data are given in [34]. This power system contains 54 generators, 9 transformers, and 186 lines. The total load of system is 4242 MW. In this study, tap settings of the transformers and the voltage limits are taken into consideration within $0.9 \mathrm{pu}-1.1 \mathrm{pu}$ and $0.94 \mathrm{pu}-1.06 \mathrm{pu}$, respectively.

The results obtained by proposed method are given in Table 6. To compare the performance of proposed method,

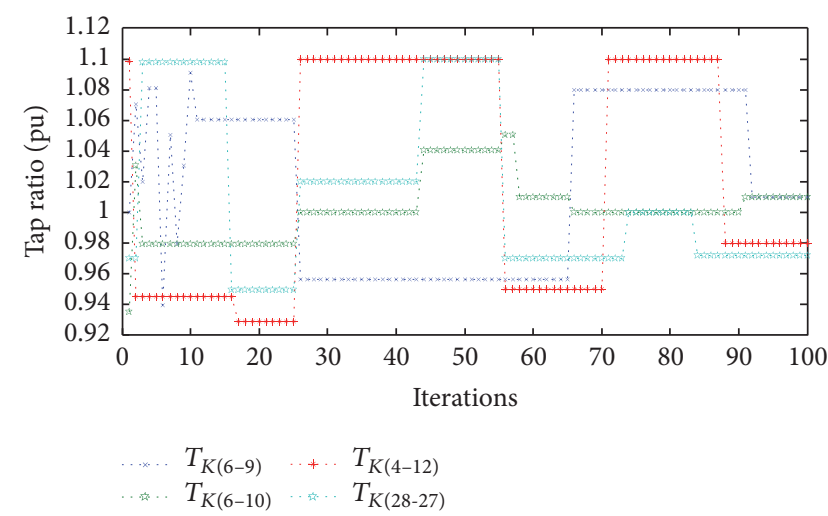

FIGURE 3: The variation of tap ratios for IEEE 30-bus power system.

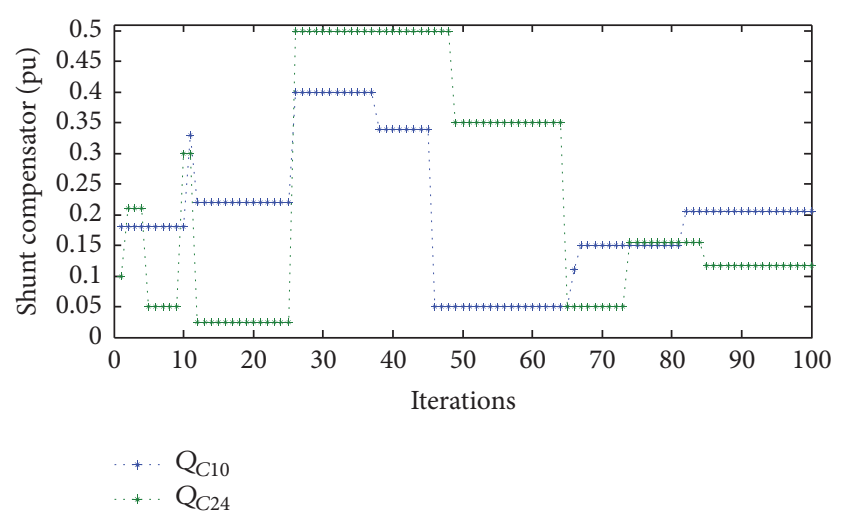

FIGURE 4: The variation of shunt compensators for IEEE 30-bus power system.

the minimum power loss obtained by different methods is also tabulated. The power loss has been reduced to 119.5500 MW. Table 6 illustrates that in this section the proposed method is more robust and effective than the other methods. Moreover, the convergence of power loss for IEEE 118-bus power system is given in Figure 5. Table 7 and Figure 6 show the tap ratios, the values of shunt compensators, the voltage, and power of generators, respectively.

6.3. Case Study on a Real Distribution Subsystem. The proposed method has been used to minimize the power loss by using reactive power optimization on Eregli Distribution Subsystem in Turkey. All data and parameter have been supplied from Eregli Branch Office of Meram Electricity Distribution Corporation.

The power subsystem considered in this paper includes 1311 buses at $0.4 \mathrm{kV}, 9$ buses at $31.5 \mathrm{kV}$, and 3 buses at $15.8 \mathrm{kV}$ and 12 branches with adjustable tab under load. First bus is selected as the slack bus, 2-9 buses are PV buses, and others are selected as PQ buses (switching capacitor banks are located on all $0.4 \mathrm{kV}$ buses). In this respect, vector dimensions become 1333 dimensions composed of nine voltage magnitude values, 12 tab settings, and 1311 switchable capacitor banks. The discrete control variables given in Table 8 are changed with step of 0.01 per unit (pu). The active power 
TABLE 6: Comparison of the power loss for IEEE 118-bus test system.

\begin{tabular}{lc}
\hline Variables & $P_{\text {loss }}(\mathrm{MW})$ \\
\hline GA & 139.16 \\
PSO & 135.64 \\
ABC & 119.6923 \\
FA & - \\
DE & 128.318 \\
hGAPSO & - \\
ESPSO & 119.5500 \\
\hline
\end{tabular}

TABle 7: Tap ratio.

\begin{tabular}{lc}
\hline Variables & ESPSO \\
\hline$T_{K(8-5)}$ & 0.9850 \\
$T_{K(26-25)}$ & 0.9900 \\
$T_{K(30-17)}$ & 0.9910 \\
$T_{K(38-37)}$ & 0.9850 \\
$T_{K(63-59)}$ & 0.9500 \\
$T_{K(64-61)}$ & 1.0000 \\
$T_{K(65-66)}$ & 0.9950 \\
$T_{K(68-69)}$ & 0.9450 \\
$T_{K(81-80)}$ & 1.0050 \\
\hline
\end{tabular}

load and reactive power load are $0.9869 \mathrm{pu}$ and $0.2242 \mathrm{pu}$, respectively, and total power losses are 1.0961 MW (note that pu values are computed on 100 MVA base).

For comparing the proposed method with GA, PSO, $\mathrm{ABC}, \mathrm{FA}$, and hGAPSO, the performance index including minimum active power losses is illustrated in Figure 7. All algorithms are run in 100 iterations and over 50 times. The results tabulated by all algorithms have been obtained after 50 times running. In addition, ESPSO has found the minimum power loss as $0.8973 \mathrm{MW}$, and all results are given in Table 9 (values of capacitor banks have not been given because of containing too many items). It can be seen that ESPSO is more effective and superior compared to other algorithms.

In order to clarify the ability of ESPSO that is statistically more robust and better than other methods, it can be shown that the minimum power loss obtained by ESPSO is smaller than all others listed and it conducts global searches accurately. After 50 times running it can be seen that the power losses are decreased from 1.0961 MW to $0.8973 \mathrm{MW}$ by the proposed ESPSO which is the highest power loss reduction. The results obtained by all algorithms handled in this paper are given below, which indicates minimum power loss, voltage magnitude of buses, and tap ratios.

\section{Conclusion}

Eagle strategy is a method of combination of global search and intensive local search for optimization. In this paper, an approach, ES with PSO based reactive power optimization method, has been implemented to a reactive power optimization problem for minimizing the power losses of 30-bus test system, 118-bus test system, and a real distribution subsystem.

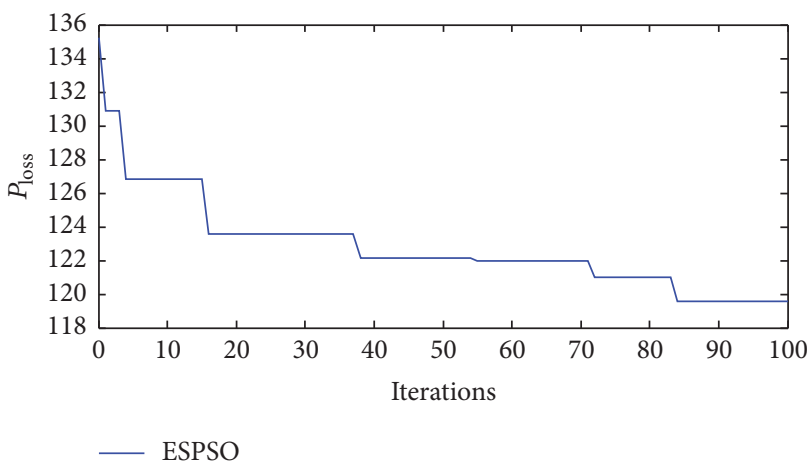

FIGURE 5: The convergence curve of power loss (MW) for 118-bus power system.

TABLE 8: The limits of control variables.

\begin{tabular}{lcc}
\hline Parameter & Min. val. $(\mathrm{pu})$ & Max. val. $(\mathrm{pu})$ \\
\hline Voltage & 0.9 & 1.1 \\
Capacitor & 0.1 & 0.61 \\
Tap position & 0.9 & 1.1 \\
\hline
\end{tabular}

It can be seen that two case studies in this paper which are performance test on benchmark functions and the minimization of power losses of various power systems may help to clarify the capability of proposed approach for optimization problem. Furthermore, ESPSO is effectively solving optimization problems and finding the optimum more successfully than the other algorithms. On the other hand, about benchmark functions performance, the proposed approach has a better performance than all other algorithms. So, it can be seen that the proposed ESPSO algorithm is influential and able to solve power loss minimization problem and may become a good candidate for other optimization problems such as reactive power dispatch, cost minimization, or multiobjective optimization.

\section{Nomenclature}

$P_{\text {loss }}:$ Total power losses

$g_{h(i, j)}:$ Conductance between nodes $i$ and $j$

$b_{h(i, j)}:$ Admittance between nodes $i$ and $j$

$\theta_{i, j}: \quad$ Angle difference between nodes $i$ and $j$

$N_{i}$ : Number of power system buses

$N_{\mathrm{slb}}:$ Number of slack buses

$N_{\mathrm{pq}}$ : Number of PQ buses

$N_{K}$ : Number of transformer branches

$N_{C}$ : Number of capacitor installed buses

$N_{G}$ : Number of generator buses

$T_{K}:$ Tap position of transformers at branches $K$

$Q_{C}$ : Shunt capacitor value

$V_{i}: \quad$ Voltage magnitude of bus $i$

$V_{j}: \quad$ Voltage magnitude of bus $j$

$V_{G}: \quad$ Voltage magnitude of each generator

$V_{L}$ : Voltage magnitude of each bus

$Q_{G}:$ The injected reactive power

$P_{G}$ : The injected active power 


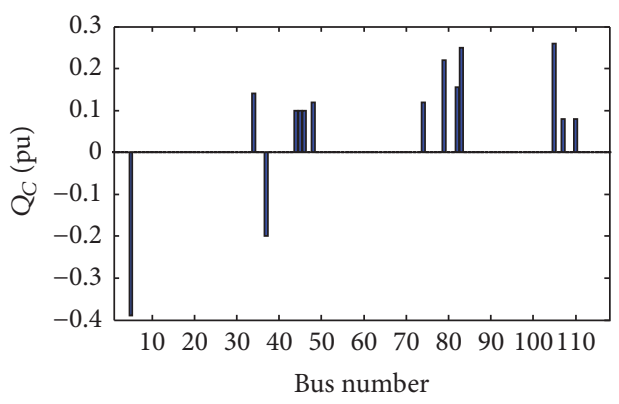

(a)

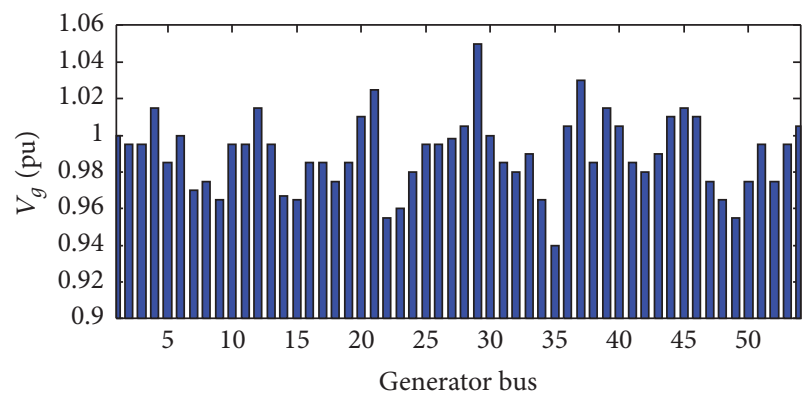

(b)

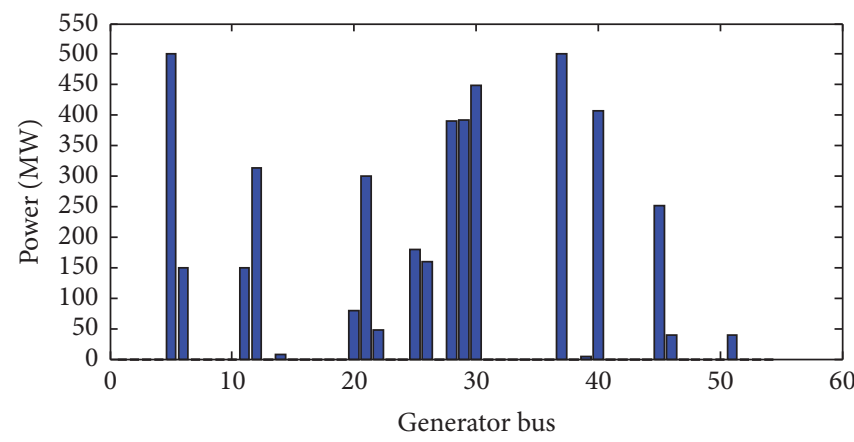

(c)

FIGURE 6: Variables of IEEE 118-bus power system: (a) value of shunt compensator (pu), (b) voltages of generators (pu), and (c) power of generators (MW).

TABLE 9: Control variables values and power loss (control variables given pu).

\begin{tabular}{|c|c|c|c|c|c|c|c|}
\hline Variables & GA & PSO & $\mathrm{ABC}$ & FA & $\mathrm{DE}$ & hGAPSO & ESPSO \\
\hline$V_{1}$ & 1.0100 & 1.0200 & 1.0400 & 1.0600 & 1.0600 & 1.0600 & 1.0600 \\
\hline$V_{2}$ & 1.0300 & 1.0400 & 1.0200 & 1.0100 & 0.9900 & 1.0300 & 1.0500 \\
\hline$V_{3}$ & 0.9800 & 1.0200 & 1.0400 & 1.0100 & 1.0600 & 1.0600 & 1.0300 \\
\hline$V_{4}$ & 1.0400 & 1.0300 & 1.0200 & 1.0400 & 0.9800 & 1.0300 & 1.0500 \\
\hline$V_{5}$ & 0.9900 & 1.0600 & 1.0400 & 0.9900 & 0.9500 & 1.0600 & 1.0200 \\
\hline$V_{6}$ & 0.9600 & 1.0600 & 1.0200 & 1.0500 & 1.0100 & 1.0300 & 1.0100 \\
\hline$V_{7}$ & 1.0600 & 1.0600 & 1.0400 & 1.0600 & 1.0100 & 1.0600 & 1.0100 \\
\hline$V_{8}$ & 1.0600 & 1.0500 & 1.0200 & 1.0500 & 0.9700 & 1.0300 & 1.0400 \\
\hline$V_{9}$ & 1.0200 & 1.0400 & 1.0000 & 1.0000 & 0.9900 & 1.0000 & 1.0500 \\
\hline$T_{1}$ & 1.07 & 1.03 & 1 & 0.98 & 1.05 & 1 & 1 \\
\hline$T_{2}$ & 1.05 & 1 & 1 & 0.98 & 1.04 & 0.97 & 0.98 \\
\hline$T_{3}$ & 1 & 1.06 & 1 & 1.04 & 1.05 & 0.97 & 1.04 \\
\hline$T_{4}$ & 1.01 & 1 & 1 & 1.01 & 1 & 0.98 & 1.01 \\
\hline$T_{5}$ & 1.03 & 1 & 1.05 & 0.97 & 0.98 & 1.01 & 1 \\
\hline$T_{6}$ & 1 & 1 & 1.03 & 1.01 & 0.98 & 1.05 & 1 \\
\hline$T_{7}$ & 0.98 & 0.97 & 1.03 & 0.99 & 0.97 & 1.04 & 0.98 \\
\hline$T_{8}$ & 0.96 & 0.98 & 0.97 & 1.03 & 1 & 0.98 & 1.03 \\
\hline$T_{9}$ & 1 & 1 & 0.98 & 0.98 & 0.98 & 1 & 0.99 \\
\hline$T_{10}$ & 0.98 & 0.98 & 0.97 & 1.04 & 1.04 & 1 & 1 \\
\hline$T_{11}$ & 0.97 & 0.96 & 1.06 & 1 & 1.05 & 1.01 & 1 \\
\hline$T_{12}$ & 0.99 & 0.07 & 1.05 & 1 & 1.01 & 1.01 & 1 \\
\hline$P_{\text {loss }}(\mathrm{MW})$ & 0.91576 & 0.91189 & 0.91758 & 0.90622 & 0.91760 & 0.90922 & 0.89730 \\
\hline
\end{tabular}




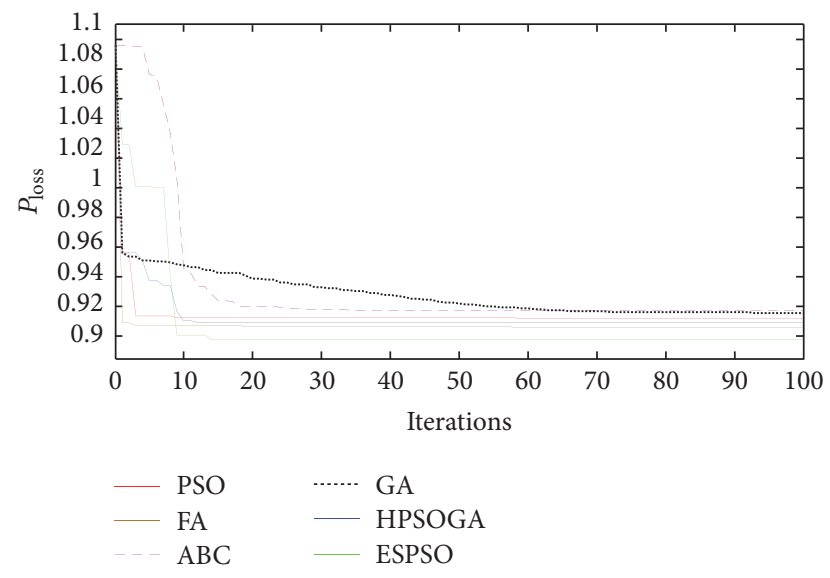

Figure 7: Convergence of active power loss (MW).

$Q_{l}: \quad$ The reactive power demanded by load

$P_{l}$ : $\quad$ The active power demanded by load

$I_{l(i, j)}$ : Transmission line current flow capacity at line between nodes $i$ and $j$

$L(s)$ : Lévy distribution function

$\Gamma(\lambda)$ : Standard gamma function

$\lambda$ : $\quad$ Gamma function parameter

$s_{0}$ : The initial step length value

$s: \quad$ Step length

$k$ : Number of iterations

$v$ : Velocity of a particle in PSO

$x$ : $\quad$ Position of a particle in PSO

pbest: Local best position

gbest: Global best

$w$ : The weight function

$r_{1}, r_{2}$ : Selected randomly in the range $[0,1]$

$c_{1}, c_{2}$ : Acceleration coefficients

$p$ : Control parameter of switching

$f$ : Objective function.

\section{Conflicts of Interest}

The authors declare that they have no conflicts of interest.

\section{References}

[1] F.-C. Lu and Y.-Y. Hsu, "Reactive power/voltage control in a distribution substation using dynamic programming," IEE Proceedings: Generation, Transmission and Distribution, vol. 142, no. 6, pp. 639-645, 1995.

[2] O. Alsaç, J. Bright, M. Prais, and B. Stott, "Further developments in LP-based optimal power flow," IEEE Transactions on Power Systems, vol. 5, no. 3, pp. 697-711, 1990.

[3] S. Granville, "Optimal reactive dispatch through interior point methods," IEEE Transactions on Power Systems, vol. 9, no. 1, pp. 136-146, 1994.

[4] K. Iba, "Reactive power optimization by genetic algorithm," IEEE Transactions on Power Systems, vol. 9, no. 2, pp. 685-692, 1994.

[5] N. Grudinin, "Reactive power optimization using successive quadratic programming method," IEEE Transactions on Power Systems, vol. 13, no. 4, pp. 1219-1225, 1998.
[6] E. Wu, Y. Huang, and D. Li, "An adaptive particle swarm optimization algorithm for reactive power optimization in power system," in Proceedings of the 8th World Congress on Intelligent Control and Automation (WCICA '10), pp. 3132-3137, Jinan, China, July 2010.

[7] X. Zhang, W. Chen, C. Dai, and A. Guo, "Self-adaptive differential evolution algorithm for reactive power optimization," in Proceedings of the 4th International Conference on Natural Computation (ICNC '08), pp. 560-564, Jinan, China, October 2008.

[8] G. Lirui, H. Limin, Z. Liguo, L. Weina, and H. Jie, "Reactive power optimization for distribution systems based on dual population ant colony optimization," in Proceedings of the 27th Chinese Control Conference (CCC '08), pp. 89-93, IEEE, Kunming, China, July 2008.

[9] H. Wei, Z. Cong, Y. Jingyan et al., "Using bacterial chemotaxis method for reactive power optimization," in Proceedings of the Transmission and Distribution Exposition Conference: IEEE PES Powering Toward the Future (PIMS '08), April 2008.

[10] B. Bhattacharyya and V. K. Gupta, "Fuzzy based evolutionary algorithm for reactive power optimization with FACTS devices," International Journal of Electrical Power and Energy Systems, vol. 61, pp. 39-47, 2014.

[11] A. Q. H. Badar, B. S. Umre, and A. S. Junghare, "Reactive power control using dynamic particle swarm optimization for real power loss minimization," International Journal of Electrical Power and Energy Systems, vol. 41, no. 1, pp. 133-136, 2012.

[12] P. R. Sujin, T. R. D. Prakash, and M. M. Linda, "Particle swarm optimization based reactive power optimization," Journal of Computing, vol. 2, pp. 73-78, 2010.

[13] S. Liu, Y. Han, Y. Ouyang, and Q. Li, "Multi-objective reactive power optimization by Modified Artificial Fish Swarm Algorithm in IEEE 57-bus power system," in Proceedings of the 6th IEEE PES Asia-Pacific Power and Energy Engineering Conference (APPEEC '14), pp. 1-5, IEEE, Hong Kong, December 2014.

[14] C. Dai, W. Chen, Y. Zhu, and X. Zhang, "Seeker optimization algorithm for optimal reactive power dispatch," IEEE Transactions on Power Systems, vol. 24, no. 3, pp. 1218-1231, 2009.

[15] A. Ozturk and S. Duman, "Determination of the optimal operating conditions in power systems using genetic algorithm," Gazi University Engineering and Architecture Faculty Journal, vol. 24, pp. 539-548, 2009.

[16] Y. Liu, L. Ma, and J. Zhang, "GA/SA/TS hybrid algorithms for reactive power optimization," in Proceedings of the Power Engineering Society Summer Meeting, pp. 245-249, Washington, DC, USA, July 2000.

[17] S. Durairaj and B. Fox, "Evolutionary computation based reactive power optimization," in Proceedings of the IET-UK International Conference on Information and Communication Technology in Electrical Sciences (ICTES '007), pp. 120-125, Dr. M.G.R. University, Chennai, India, December 2007.

[18] S. Cheng and M.-Y. Chen, "Multi-objective reactive power optimization strategy for distribution system with penetration of distributed generation," International Journal of Electrical Power and Energy Systems, vol. 62, pp. 221-228, 2014.

[19] Q. Liu and J. Yue, "An improved mind evolutionary algorithm for reactive power optimization," in Proceedings of the 32nd Chinese Control Conference (CCC '13), pp. 8048-8051, Xi'an, China, July 2013.

[20] R. Lukomski and K. Wilkosz, "Optimization of reactive power flow in a power system for different criteria: stability problems," 
in Proceedings of the 8th International Symposium on Advanced Topics in Electrical Engineering (ATEE '13), pp. 1-6, Bucharest, Romania, May 2013.

[21] X.-S. Yang, S. Deb, and S. Fong, "Metaheuristic algorithms: optimal balance of intensification and diversification," Applied Mathematics and Information Sciences, vol. 8, no. 3, pp. 977-983, 2014.

[22] X.-S. Yang, S. Deb, and X. He, "Eagle strategy with flower algorithm," in Proceedings of the 2nd International Conference on Advances in Computing, Communications and Informatics (ICACCI '13), pp. 1213-1217, IEEE, Mysore, India, August 2013.

[23] R. Jia and D. He, "Artificial bee colony algorithm with two-stage eagle strategy," in Proceedings of the 9th International Conference on Computational Intelligence and Security (CIS '13), pp. 16-20, IEEE, Leshan, China, December 2013.

[24] K. Ayan and U. Kılıç, "Artificial bee colony algorithm solution for optimal reactive power flow," Applied Soft Computing, vol. 12, no. 5, pp. 1477-1482, 2012.

[25] A. H. Gandomi, X.-S. Yang, S. Talatahari, and S. Deb, “Coupled eagle strategy and differential evolution for unconstrained and constrained global optimization," Computers and Mathematics with Applications, vol. 63, no. 1, pp. 191-200, 2012.

[26] X. S. Yang, S. Deb et al., "Eagle strategy using Lévy walk and firefly algorithms for stochastic optimization," in Nature Inspired Cooperative Strategies for Optimization (NICSO 2010), J. R. Gonzalez, D. A. Pelta, C. Cruz, G. Terrazas, and N. Krasnogor, Eds., vol. 284, pp. 101-111, Springer, 2010.

[27] W. Zhang and Y. Liu, "Reactive power optimization based on PSO in a practical power system," in Proceedings of the IEEE Power Engineering Society General Meeting, pp. 239-243, June 2004.

[28] N. K. Sharma, D. Suresh Babu, and S. C. Choube, "Application of particle swarm optimization technique for reactive power optimization," in Proceedings of the International Conference on Advances in Engineering, Science and Management (ICAESM '12), pp. 88-93, IEEE, Nagapattinam, India, March 2012.

[29] W. Zhang and Y. T. Liu, "Multi-objective reactive power and voltage control based on fuzzy optimization strategy and fuzzy adaptive particle swarm," International Journal of Electrical Power and Energy Systems, vol. 30, no. 9, pp. 525-532, 2008.

[30] H. Deenadhayalan, "Real power loss minimization using firefly algorithm," International Journal of Advanced Information and Communication Technology, vol. 1, no. 8, pp. 677-682, 2014.

[31] M. Varadarajan and K. S. Swarup, "Differential evolutionary algorithm for optimal reactive power dispatch," International Journal of Electrical Power and Energy Systems, vol. 30, no. 8, pp. 435-441, 2008.

[32] K. Lenin, B. R. Reddy, and M. S. Kalavathi, "A new improved GA and PSO combined hybrid algorithm (HIGAPSO) for solving optimal reactive power dispatch problem," Journal of Industrial and Intelligent Information, vol. 2, no. 3, pp. 205-209, 2014.

[33] K. Y. Lee, Y. M. Park, and J. L. Ortiz, "A united approach to optimal real and reactive power dispatch," IEEE Transactions on Power Apparatus and Systems, vol. 104, no. 5, pp. 1147-1153, 1985.

[34] Power system data for IEEE 118-bus test system, 2014, http:// www.ece.ubc.ca/ hameda/download_files/case118.m. 


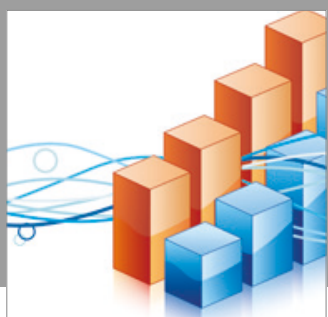

Advances in

Operations Research

vatersals

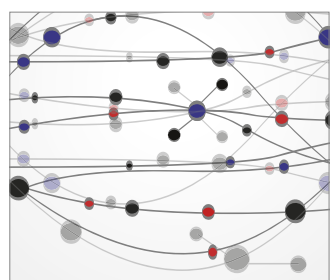

\section{The Scientific} World Journal
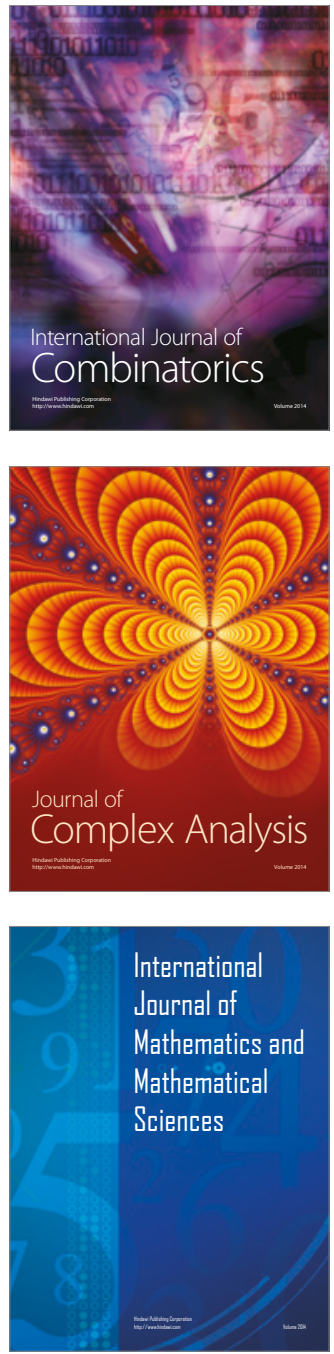
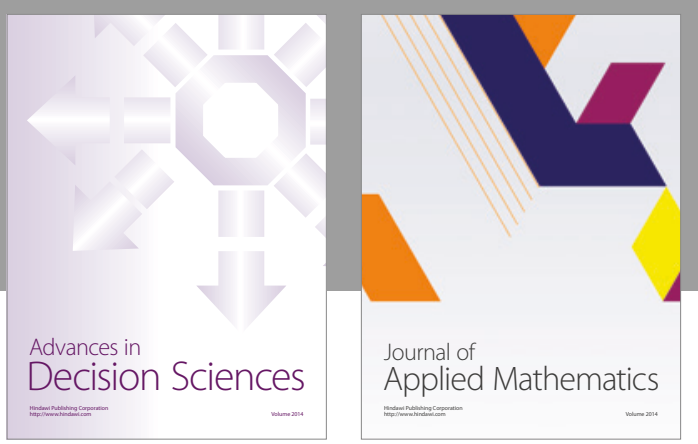

Algebra

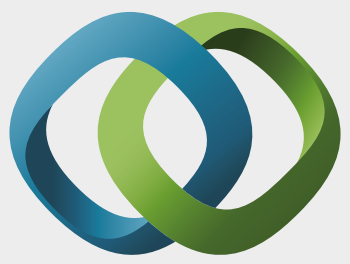

\section{Hindawi}

Submit your manuscripts at

https://www.hindawi.com
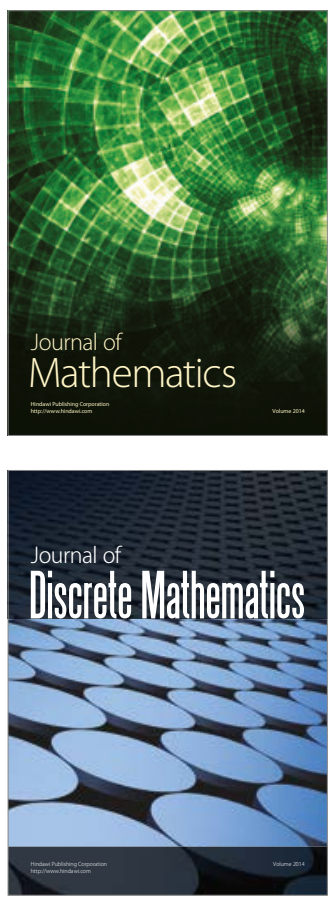

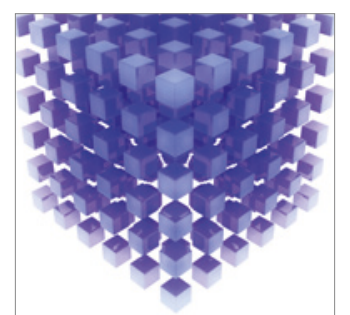

Mathematical Problems in Engineering
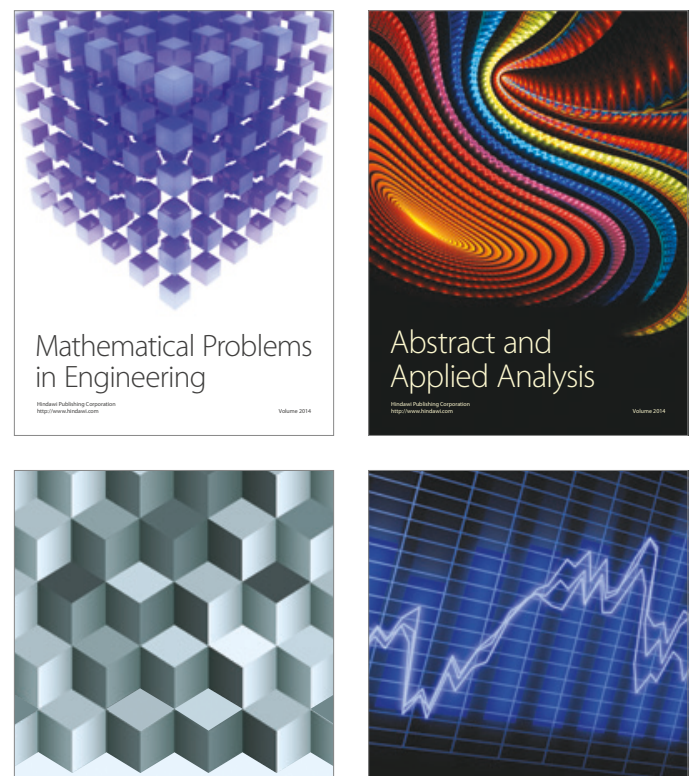

Journal of

Function Spaces

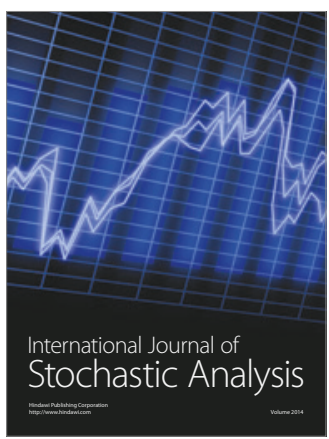

Probability and Statistics
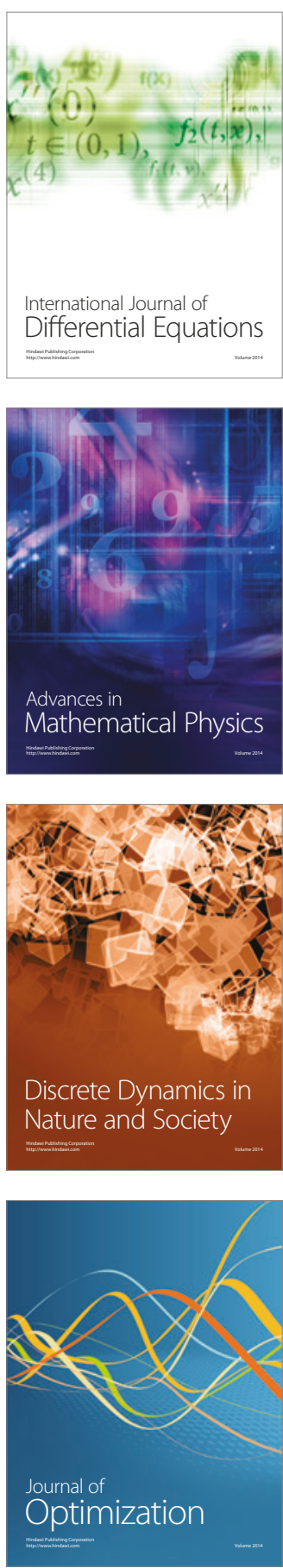